\title{
Discrimination and Prediction of Pork Freshness by E-nose
}

\author{
Xuezhen Hong and Jun Wang* \\ Department of Biosystem Engineering, Zhejiang University, Hangzhou 310058, P.R. China \\ \{xzhong, jwang\}@zju.edu.cn
}

\begin{abstract}
An electronic nose (e-nose) was used to establish a freshness evaluation model for pork. A pre-experiment was performed to acquire optimum parameters ( $10 \mathrm{~g}$ sample mass with 5 min headspace-generation time in $500 \mathrm{~mL}$ vial) for later e-nose detection of pork. Responding signals of the enose were extracted and analyzed. Linear Discriminant Analysis (LDA) results showed that the e-nose could classify pork with different storage time (ST) well. Back Propagation Neural Network (BPNN) was performed to predict the ST, and the results showed that $97.14 \%$ of the predicting set (with $95.71 \%$ of the training set) was classified correctly; and Multiple Linear Regression (MLR) was used to predict the sensory scores, with the results showing that the correlation coefficients $\left(R^{2}=0.9848\right)$ between the e-nose signals and the sensory scores was high. These results prove that e-nose has the potential of assessing pork freshness.
\end{abstract}

Keywords: Electronic nose, Pork freshness, Prediction, Back Propagation Neural Network, Multiple Linear Regression.

\section{Introduction}

Due to its high nutritional value and tasty taste, the consumption of pork has been increasing dramatically during the last decades. However, pork is highly susceptible to spoilage and contamination by micro-organisms. The main ingredients of pork are water, protein, fat and a small amount of carbohydrates. During the storage, the ingredients will be decomposed by enzymes and bacteria, producing odor: the protein will be decomposed into ammonia, hydrogen sulfide, ethyl mercaptan, etc.; the fat will be decomposed into aldehydes and aldehyde acids odor; the carbohydrates will be decomposed into alcohols, ketones, aldehydes, and carboxylic acid gases [1]. The odor gets more and more intense with the decrease of pork freshness. Consumption of spoilage pork could cause serious health hazards [2]. Thus, it is necessary that a rapid and accurate detection system be developed for microbiologically spoiled or contaminated pork [3-4].

At present, there are mainly three traditional methods in the meat industry to detect pork freshness: sensory evaluation based on the texture, color, organization

* Corresponding author. 
status, viscidity and odor of the pork [5]; detection of the total volatile basic nitrogen (TVBN) [6]; and aerobic plate counts of the pork samples using standard protocols (FDA-US Food and Drugs Administration, 1998) [7]. The first method provides immediate quality information but suffers from some disadvantages, for example, the subjective nature of the assessment. Furthermore, errors may arise from fatigue of panelists and low threshold concentrations of stale odor compounds may not be perceived [8]; The latter two methods are objective, but destructive, complicated and time consuming. Moreover, they are just used to analyze one or two specialized components instead of giving the whole information of pork quality. Consequently, these traditional methods are unsuitable for fast and on-line application in pork industry.

Electronic nose (e-nose), also known as artificial olfactory, is a simulation of biological functions to identify some simple or complex odor [9-10]. A typical enose system contains a selective chemical sensor array, a signal processing subsystem and a pattern recognition subsystem. The sensors in the sensor array are sensitive to different substances. For example, some sensors can discern ammonia and some can discern aldehydes. Thus the whole sensor array can discern complex odor. Instead of detecting one or two components of the substances, the e-nose extracts the whole information for identification. In the last decade, a few researchers have been studying the potential of using e-nose as a non-destructive method for food detection. García et al. [11] used a metal oxide semiconductor thin-film sensors based electronic nose to characterize and classify four types of red wines of the same variety of grapes which came from the same cellar. Two pattern recognition methods: Principal Component Analysis (PCA) and Probabilistic Neuronal Network (PNN) were performed, and the results showed that electronic nose was able to identify the wine well; Torri, Sinelli, and Limbo [12] used a commercial electronic nose to monitor the freshness of minimally processed fruit (packaged pineapple slices) during storage. The samples were stored at three different temperatures $\left(4-5,7-8\right.$, and $\left.15-16{ }^{\circ} \mathrm{C}\right)$ for $6-10$ days. After a continuous monitoring of the headspace around the fruit, the result showed that the fruit freshness was maintained for about 5 days at $4{ }^{\circ} \mathrm{C}, 2$ days at $7.6{ }^{\circ} \mathrm{C}$ and 1 day at $16{ }^{\circ} \mathrm{C}$.

In the previous research, most sensor arrays used in e-nose are Metal-Oxide Semiconductors (MOS). However, seldom information about the quality assurance of the e-nose performance has been given regarding sensor drift and humidity (MOS sensors used in e-nose are water sensitive), so it is impossible to rule out the fact that their research results may be significantly affected by either a day to day sensor drift of the sensor system, or the fact that the temporal changes observed in the sensor reading is due to the fact that the sensors perceive increasingly proportions of humidity due to increase in water vapor during storage. Moreover, few papers have mentioned the study of optimum experimental parameters, and the recognition models used are just focused on discrimination rather than prediction. In most cases, only e-nose was used, with no other experiments carried out. So even if we could predict the storage time of the food, we still can't precisely identify its freshness degree, since we don't have other indexes for cross-reference. 
In this research, two experiments were conducted: e-nose detection and sensory evaluation. The main objective of this research is to evaluate the capacity of an electronic nose to classify pork samples stored for different time, as well as to predict their storage time and sensory scores. As for the e-nose detection, the sensor drift and humidity problem were taken care of by controlling the environment parameters (temperature and humidity) and by choosing calibrated data as the initial data. A preexperiment was conducted to study the effects of headspace-generation time and pork sample mass on the response of e-nose performance. The optimum experimental parameters of headspace-generation time and sample mass were determined after employing Multivariate Analysis of Variance (MANOVA) and One-Way Analysis of Variance (ANOVA), and the later e-nose experiment was taken under the optimum experimental parameters. Linear Discriminant Analysis (LDA) and Back Propagation Neural Network (BPNN) were employed to observe if the e-nose could classify the pork samples stored for 0-6 d well, as well as to predict the storage time; and Multiple Linear Regression (MLR) was employed to build the prediction model between e-nose data and sensory scores.

\section{Materials and Method}

\subsection{Sample Preparation}

Fresh lean pork samples were purchased twice: the first purchase was for the preexperiment, and the second purchase was for the discrimination of pork samples stored for 0-6 d. For both of the time, all the samples were obtained from the same parts of pigs and the same supplier in the local farmers' market (30.26 N, 120.19 $\mathrm{E}$, Zhejiang province, China) $4 \mathrm{~h}$ after been killed, and were minced immediately on the spot. All the samples were packaged immediately using polystyrene base trays and were covered with commercial food grade polymer wraps before being transported to the lab. The samples were stored at $10{ }^{\circ} \mathrm{C}$ in the fridge before detection, except the ones detected on the first day (marked as day 0).

\subsection{Electronic Nose System}

The experiment was performed with a portable electronic nose (PEN2, Airsense Analytics, GmBH, Schwerin, Germany) (Fig. 1), which is consisted of an autosampling apparatus that is exposed to the volatiles, a sensor array, and pattern recognition software that is ran on a computer. The sensor array is composed of ten MOS. A description of the ten MOS is given in Table 1.

The operating process is based on Win Muster V1.6 software. There are two kinds of data obtained from the e-nose, one is $R$ (the resistance value of the sensors when the sample gas flow through them), the other is $G / G_{0}$, where $G$ and $G_{0}$ are the conductivities of the sensor when exposed to the sample gas and the zero gas, respectively. The $G / G_{0}$ value is more reliability cause it could avoid sensor drift in some degree, so in this study, the $G / G_{0}$ value is chosen as the initial data. 


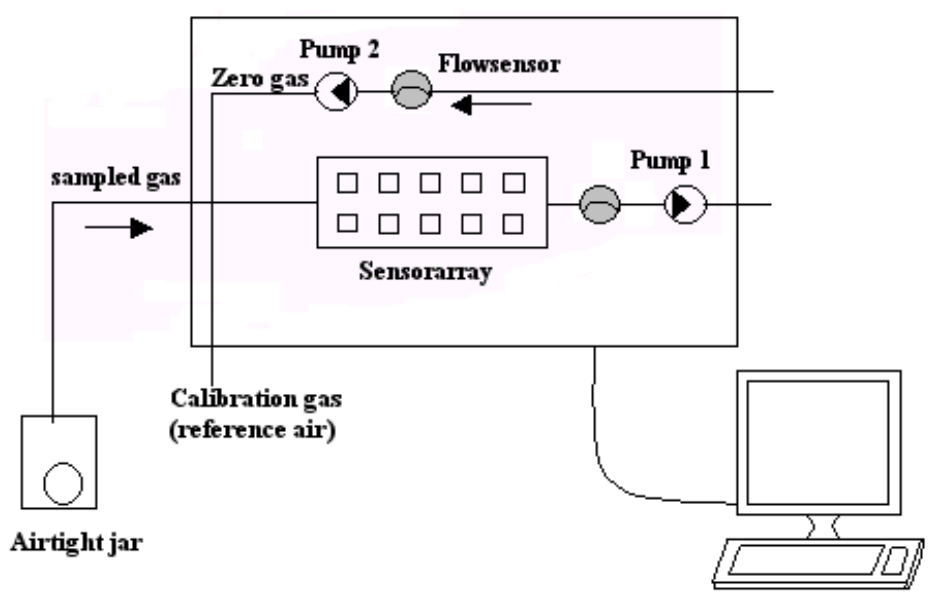

Fig. 1. Schematic diagram of the electronic nose (e-nose) measurements

Table 1. Sensors used and their object substances in PEN2

\begin{tabular}{lll}
\hline Array number & Sensor & Substances for Sensing \\
\hline MOS 1 & W1C & Aromatics \\
MOS 2 & W5S & Nitrogen oxides \\
MOS 3 & W3C & Ammonia, aromatic molecules \\
MOS 4 & W6S & Hydrogen \\
MOS 5 & W5C & Methane, propane, and aliphatic non-polar molecules \\
MOS 6 & W1S & Methane \\
MOS 7 & W1W & Sulfur-containing organics \\
MOS 8 & W2S & Broad alcohols \\
MOS 9 & W2W & Aromatics, sulfur- and chlorine-containing organics \\
MOS 10 & W3S & Methane and aliphatics \\
\hline
\end{tabular}

\subsection{Experimental Procedure}

Sensory Evaluation. A sensory evaluation of pork is a method for description of the quality in an objective way. A descriptive method in accordance with GB/T 5009.44-2003 [13] was carried out at the Department of Biosystem Engineering and Food Science by a selected and trained sensory panel consisting of 8 assessors. All assessors were familiar with pork characters and descriptive analysis procedure. Scoring standard is given in Table 2. 
Table 2. Sensory evaluation standards

\begin{tabular}{|c|c|c|c|}
\hline \multirow{2}{*}{ Parameters ${ }^{-}$} & \multicolumn{3}{|c|}{ Scores } \\
\hline & 5 & 3 & 1 \\
\hline Color & $\begin{array}{l}\text { Muscle: shiny, red, uniform } \\
\text { Fat : white }\end{array}$ & $\begin{array}{l}\text { Muscle: a little dark red } \\
\text { Fat : lack of luster }\end{array}$ & $\begin{array}{l}\text { Dark color with } \\
\text { sign of depravity }\end{array}$ \\
\hline Viscosity & Slightly dry or moist & $\begin{array}{l}\text { Sticky or dry } \\
\text { moist new cut section }\end{array}$ & High viscosity \\
\hline Elasticity & $\begin{array}{l}\text { Instantly and completely } \\
\text { recover from acupressure }\end{array}$ & $\begin{array}{l}\text { Slowly and incompletely } \\
\text { recover from acupressure }\end{array}$ & Low elastic \\
\hline Flavor & Normal & Ammonia or sour odor & Strong odor \\
\hline
\end{tabular}

E-nose Sampling Procedure. The concentration of the volatile gas is affected by the mass and headspace-generation time of the samples [14], so a set of pre-experiments were performed to determine the optimum experimental parameters. Three main factors were considered: mass of the pork samples (M: 10 and $25 \mathrm{~g}$ ), storage time (ST: 0 and 1day) and headspace-generation time (HGT: 5, 15 and $25 \mathrm{~min}$ ). The samples stored for 0 and 1 day were divided into six groups respectively, marked as 10-5, 10$15,10-25,25-5,25-15,25-25$. The number format was mass - headspace-generation time, for example, the 10-5 group means $10 \mathrm{~g}$ sample mass with 5 min headspacegeneration time. The multifactor pre-experiment was conducted with seven replicates of each group (Table 3). After acquiring the optimum experimental parameters, the pork samples stored for 0-6 d (15 replicates each day) were detected by e-nose under such parameters.

Each pork sample was placed in a $500 \mathrm{~mL}$ airtight glass vial that was sealed with plastic wrap. The glass vial was closed for a certain time (headspacegeneration time) to collect the volatiles from the pork sample. During the measurement process, the headspace gaseous compounds were pumped into the sensor array at a constant flow rate of $50 \mathrm{~mL} \mathrm{~min}{ }^{-1}$ through Teflon tubing connected to a needle in the plastic wrap, making the ratio of conductance of each sensor change. The measurement phase lasted for $65 \mathrm{~s}$, which was long enough for the sensors to reach stable signal values. The signal data from the sensors were collected by the computer once per second during the measurements. When the measurement process was complete, the acquired data were stored for later mathematic analysis. After each measurement, zero gas (air filtered by active carbon) was pumped into the sample gas path from the other port of the instrument for $50 \mathrm{~s}$ (flush time). In case of sensor pollution which could cause sensor drift, after all the measurements were done every day, nitrogen gas was pumped into the sample gas path to clear the sensor array. All the measurements were carried out at a temperature of $20{ }^{\circ} \mathrm{C} \pm 1{ }^{\circ} \mathrm{C}$ and $50 \%$ to $60 \%$ relative humidity (controlled by air-conditioning). 
Table 3. Pre-experiment method

\begin{tabular}{ccll}
\hline Storage time/ d & Mass/g & Headspace-generation time/ min & Number of replications \\
\hline 0 & 15 & 5 & 7 \\
0 & 15 & 15 & 7 \\
0 & 15 & 25 & 7 \\
0 & 25 & 5 & 7 \\
0 & 25 & 15 & 7 \\
0 & 25 & 25 & 7 \\
1 & 15 & 5 & 7 \\
1 & 15 & 15 & 7 \\
1 & 15 & 25 & 7 \\
1 & 25 & 5 & 7 \\
1 & 25 & 15 & 7 \\
1 & 25 & 25 & 7 \\
\hline
\end{tabular}

\subsection{Statistical Analysis Methods}

LDA is a widely used statistic method. Similar to PCA, it is also a linear combination of the original variable to construct a discriminant function [15]. Compared with PCA, the LDA method can notice the distribution of points in the same category and the distance between them. It maximizes the variance between categories and minimizes the variance within categories to improve the resolution of classes [16]. The graphical view of LDA analysis is similar to a PCA display.

BPNN has been a widely-used method for e-nose [17-18]. BPNN can be described as a non-linear projection between the input vectors and output vectors. A typical BPNN model includes the input layer, the hidden layer (one layer or more) and the output layer. The dimensions of the input and output layer are usually decided by the dimensions of the input and output vectors, respectively, while the dimensions of the hidden layer is usually decided by try and error methodology. While determining the suitable network topology, the network processed the inputs and compared the resulting outputs against the desired outputs. Errors were then propagated back through the system, causing the system to adjust the weights that control the network [19]. This process continued over and over until the error matched the training goal error.

MLR analysis is a common method used in quantitative analysis. Equations relating the dependent variable behavior to the descriptors are developed with the following form: contribution, with numbers enclosed in parentheses and set on the right margin. 


$$
Y_{i}=\beta_{0}+\beta_{i} X_{i},(i=1,2,3, \ldots, \mathrm{N}) .
$$

Where $Y_{i}$ is an independent variable; $\beta_{0}$ is the intercept and $\beta_{i}$ are the regression coefficients of the independent variables $X_{i}$; and $\mathrm{N}$ is the number of independent variables.

ANOVA is a method of portioning variability into identifiable sources of variation and the associated degree of freedom in an experiment. The frequency test $(F$-test) is utilized in statistics to analyze the significant effects of the parameters, which form the quality characteristics [20]. MANOVA is a generalized form of univariate ANOVA. It is used when there are two or more dependent variables. It helps to answer: 1. do changes in the independent variable(s) have significant effects on the dependent variables; 2 . what are the interactions among the dependent variables and 3. among the independent variables [21].

The data processing method LDA was performed in WinMuster, which is combined in the e-nose software; (M)ANOVA and MLR were performed in SAS software, and BPNN was proceeded using the network toolbox in MATLAB R2008a.

\section{Results and Discussions}

\subsection{Discussions of Sensory Evaluation Results}

Sensory evaluation result is shown in Fig. 2. The sensory scores of color, elasticity, viscosity and odor attributes decreased as the storage time increased. It is also noticeable that both of the curves had declined quickly since the third day, while in the first 3 days, the total score curve only declined slightly, and the odor score nearly kept the same. This manifested that the pork sample didn't corrupt until the third day. So in the first 3 days, it remained fresh, with its appearance or odor change little.

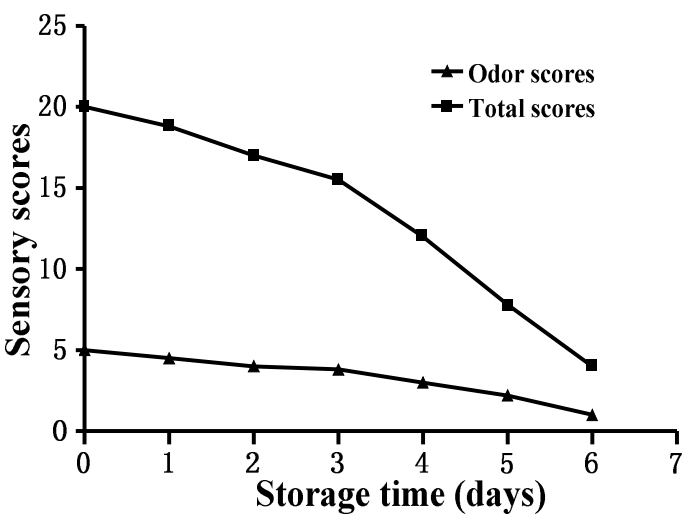

Fig. 2. Sensory evaluation result 


\subsection{Discussions of Pre-experiment Results}

Responding Curves of E-nose. Fig. 3 shows two typical responding curves of the ten sensors during measurement of a pork sample in day 0 (Fig. 3a) and day 1 (Fig. 3b), respectively. Each curve represents a sensor's ratio of conductance $\left(G / G_{0}\right)$, which increased in the first few seconds and finally stabilized at about the $60^{\text {th }} \mathrm{s}$. Compare Fig. 3a and Fig. 3b, the ratio of conductance values of all the sensors increased with the storage time of pork. It is also noticeable that the sensor MOS 2, which is sensitive to nitrogen oxides (one of the main odor in pork putrefaction), increased most observably with storage time, with its $G / G_{0}$ value differing significantly by the storage time. This indicates that it is potential to monitor pork freshness by e-nose.
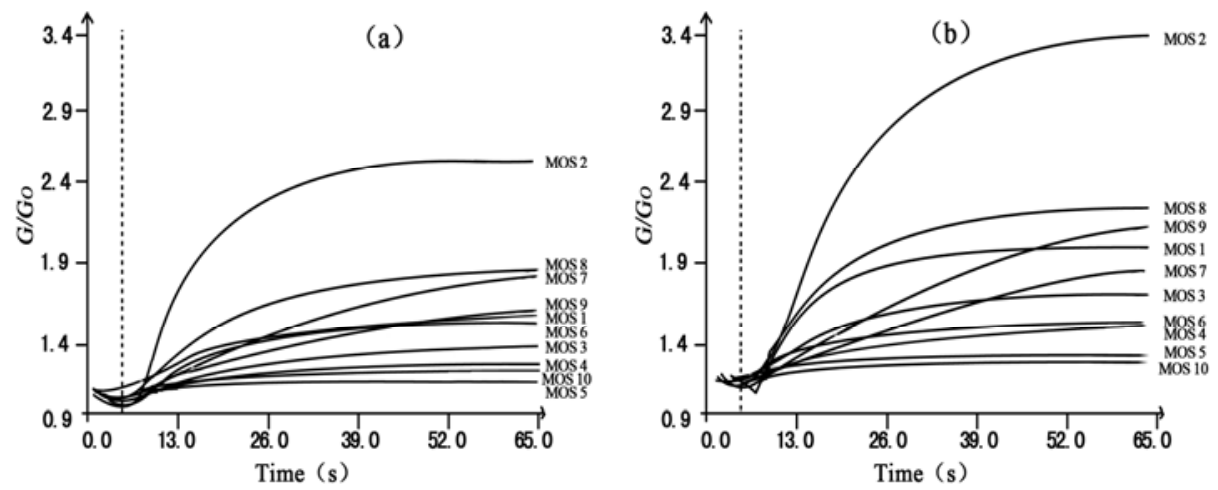

Fig. 3. Responding curves of sensors for fresh pork samples: (a): stored for 0 day; (b): stored for 1 day

\section{Optimum Experimental Parameters.}

Multivariate Analysis of Variance Result. A 3-factors analysis of variance was performed to acquire optimum experimental parameters. The three factors are sample mass (M), headspace-generation time (T) and storage time (ST).

Table 4. MANOVA (factors are storage time, mass, and headspace-generation time)

\begin{tabular}{llllll}
\hline Different Source & Sum of Squares & Degree of Freedom & Mean Squares & $F$ & Sig. \\
\hline $\mathrm{M}$ & 17.880 & 1 & 17.880 & 172.231 & 0.000 \\
$\mathrm{~T}$ & 8.043 & 2 & 4.021 & 38.736 & 0.000 \\
$\mathrm{ST}$ & 22.875 & 1 & 22.875 & 220.338 & 0.000 \\
$\mathrm{M} \times \mathrm{T}$ & 0.890 & 2 & 0.445 & 4.288 & 0.017 \\
$\mathrm{M} \times \mathrm{ST}$ & 0.145 & 1 & 0.145 & 1.393 & 0.241 \\
$\mathrm{~T} \times \mathrm{ST}$ & 0.217 & 2 & 0.108 & 1.044 & 0.357 \\
$\mathrm{M} \times \mathrm{T} \times \mathrm{ST}$ & 0.678 & 2 & 0.339 & 3.267 & 0.043 \\
\hline
\end{tabular}


MANOVA was performed to see how these factors affected the response of e-nose. The MANOVA results are summarized in Table 4, where the magnitudes of the $F$-values indicate the relative importance of the factors to some extent. As is shown in Table 4, M, T, and ST all have very significant effect on the response of the e-nose respectively (Sig. $<0.001$ ). The interaction of $\mathrm{M} \times \mathrm{T}$, and $\mathrm{M} \times \mathrm{T} \times \mathrm{ST}$ also have a significant effect on the response of the e-nose (Sig. $<0.05)$. It can also be observed that the ST has the highest $F$-value, which means the e-nose signals are differed between samples with different storage time. This proves the feasibility of using the e-nose to distinguish pork freshness. The mass factor has the second highest $F$-value, next is the headspace-generation time, suggesting that it is very important to determine the sample mass and the headspace-generation time.

One-Way Analysis of Variance Result. ANOVA (the factor is storage time) was applied for group 10-5, 10-15, 10-25, 25-5, 25-15, 25-25 and got each group a $F$-value, respectively. The results of six groups are summarized in Table 5, which shows that all the groups have very significant effects on the response of e-nose, and the combination 10-5 has the highest $F$-value. This means that when the mass is $10 \mathrm{~g}$ and the headspace-generation time is $5 \mathrm{~min}$, the e-nose has the most obvious difference in its responding values towards samples with different storage time.

Table 5. $F$-values of six combinations

\begin{tabular}{lll}
\hline Combinations & $F$-value & Sig. \\
\hline $10-5$ & 209.883 & 0.000 \\
$10-15$ & 44.131 & 0.000 \\
$10-25$ & 66.676 & 0.000 \\
$25-5$ & 89.451 & 0.000 \\
$25-15$ & 159.226 & 0.000 \\
$25-25$ & 55.049 & 0.000 \\
\hline
\end{tabular}

Therefore, in this research, the optimum parameters are $10 \mathrm{~g}$ pork sample with $5 \mathrm{~min}$ headspace-generation time.

Discrimination Power (DP). DP is another index used to observe the magnitude of difference among samples [22]. All combinations with different mass and headspacegeneration time (as was described before) were applied the DP analysis and got a value respectively (listed in Table 6). The number format is storage time - mass - headspace-generation time. For example, 0-10-5 means stored 0 day and $10 \mathrm{~g}$ sample mass with $5 \mathrm{~min}$ headspace-generation time. As is shown in Table 6, the combination 10-5 has the highest DP value, which means the e-nose response of this combination has the most obvious difference between day 0 and day 1 . This result is the same with the ANOVA result. 
Table 6. Results of DP test of six combinations

\begin{tabular}{lllllll}
\hline & $1-10-5$ & $1-10-15$ & $1-10-25$ & $1-25-5$ & $1-25-15$ & $1-25-25$ \\
\hline $0-10-5$ & 0.967 & & & & & \\
$0-10-15$ & & 0.555 & & & & \\
$0-10-25$ & & 0.753 & & & \\
$0-25-5$ & & & 0.582 & & \\
$0-25-15$ & & & & 0.852 & \\
$0-25-25$ & & & & & 0.448 \\
\hline
\end{tabular}

\subsection{Discussion of E-nose Detection Results}

The pork samples stored for 0-6 days (15 replications of each day) were detected by e-nose under the optimum experimental parameters, and the signals of e-nose were analyzed by multiple data analysis methods.

Linear Discriminant Analysis (LDA). For all 105 pork samples ( 7 storage time $\times 15$ duplicates), the response signal values of the e-nose at the $60^{\text {th }} \mathrm{s}$ were extracted and analyzed by LDA. The results are shown in Fig. 4, which is a two-dimensional spatial plot defined by two discriminant functions. The first discriminant function (LD 1) explains $64.70 \%$ of the total variance, and the second discriminant function (LD 2) explains $23.72 \%$ of the total variance. The total contribution rate is $88.42 \%$, which means these two reflect $88.42 \%$ of the original information.

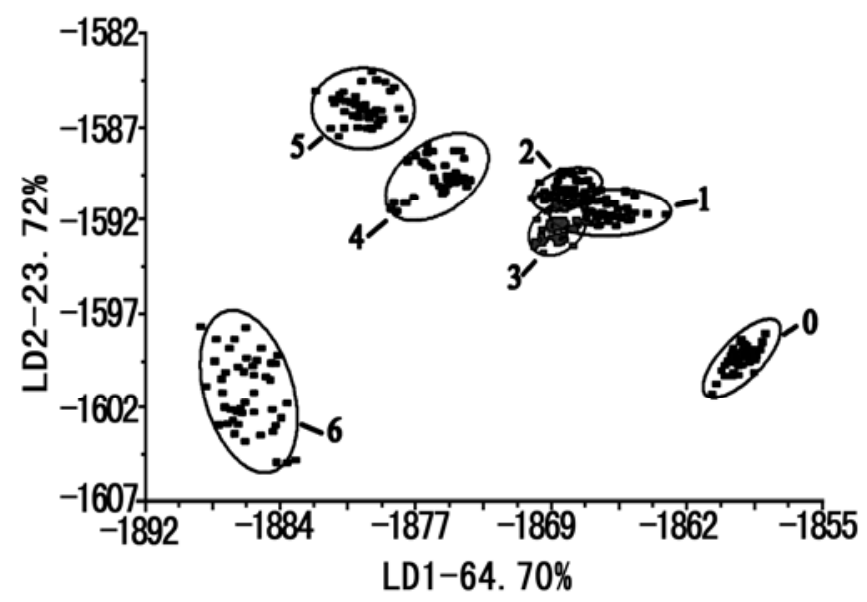

Fig. 4. LDA analysis of pork stored for 0-6 days 
As is shown in Fig. 4, the samples stored for 1-3 days are very close to each other. This may be explained as follows: in the first 0-3 days, fresh pork stored at $10{ }^{\circ} \mathrm{C}$ in the fridge still remained fresh, and the change in their volatile gases was subtle so the e-nose could not notice the difference well. Thus, the data that e-nose extracted was similar to each other and concentrated. However, freeze \& unfreeze could cause cell damage and affect the quality of pork, so the samples stored 0 day, which were taken for experiment directly without being stored in the fridge, are discriminated from those stored in fridge for 1-3 days. It is also noticeable that the samples stored 6 days are obviously discriminated. In general, except a little overlapped among the samples stored 1-3 days, all the samples can be clearly divided into seven regions according to their storage time.

Back Propagation Neural Network (BPNN). In this study, a 30-7-7-7 BPNN model was applied for the storage time prediction of the pork samples stored 0-6 days. Three eigenvalues (the 20th s, 40th s and 60th s value) of every sensor signal were adopted, and then all $30(3 \times 10)$ signal values were used as the input vector of the BPNN. The output vector was designed seven-dimensional in accordance with the seven storage days, and the training goal error was set as 0.001 . After many trials, the hidden layer was decided and the network topology was designed as 30-7-7-7.

All the 105 samples were divided into two groups: 70 samples for the training set (10 samples of each group were randomly chosen), and 35 samples for the predicting set. So the input layer for the training set and the predicting set was a $70 \times 30$ matrix ( 3 eigenvalues of each sensor) and a $35 \times 30$ matrix, respectively. The results were shown in Table 7 and Table 8.

The total identification rate of the simulated results for the training set was $95.71 \%$, and the total identification rate for the predicting set was $97.14 \%$. It should be noticed that neither the predicting set nor the training set could correctly discriminate the samples stored between 2 and 3 days.

Table 7. BP results of the original data in the training set

\begin{tabular}{|c|c|c|c|c|c|c|c|c|c|c|}
\hline \multirow[t]{2}{*}{ ST } & \multirow[t]{2}{*}{ NS } & \multicolumn{7}{|c|}{ Recognition results } & \multirow{2}{*}{$\begin{array}{l}\text { Identification rate } \\
\text { of each day }\end{array}$} & \multirow{2}{*}{$\begin{array}{l}\text { Identification } \\
\text { rate of all days }\end{array}$} \\
\hline & & 0 & 1 & 2 & 3 & 4 & 5 & 6 & & \\
\hline 0 & 10 & 10 & & & & & & & $100 \%$ & \\
\hline 1 & 10 & & 10 & & & & & & $100 \%$ & \\
\hline 2 & 10 & & & 8 & 2 & & & & $80 \%$ & \\
\hline 3 & 10 & & & 1 & 9 & & & & $90 \%$ & $95.71 \%$ \\
\hline 4 & 10 & & & & & 10 & & & $100 \%$ & \\
\hline 5 & 10 & & & & & & 10 & & $100 \%$ & \\
\hline 6 & 10 & & & & & & & 10 & $100 \%$ & \\
\hline
\end{tabular}


Table 8. BP results of the original data in the predicting set

\begin{tabular}{|c|c|c|c|c|c|c|c|c|c|c|}
\hline \multirow[t]{2}{*}{ ST } & \multirow[t]{2}{*}{ NS } & \multicolumn{7}{|c|}{ Recognition results } & \multirow{2}{*}{$\begin{array}{l}\text { Identification rate } \\
\text { of each day }\end{array}$} & \multirow{2}{*}{$\begin{array}{l}\text { Identification } \\
\text { rate of all days }\end{array}$} \\
\hline & & 0 & 1 & 2 & 3 & 4 & 5 & 6 & & \\
\hline 0 & 5 & 5 & & & & & & & $100 \%$ & \\
\hline 1 & 5 & & 5 & & & & & & $100 \%$ & \\
\hline 2 & 5 & & & 4 & 1 & & & & $80 \%$ & \\
\hline 3 & 5 & & & & 5 & & & & $100 \%$ & $97.14 \%$ \\
\hline 4 & 5 & & & & & 5 & & & $100 \%$ & \\
\hline 5 & 5 & & & & & & 5 & & $100 \%$ & \\
\hline 6 & 5 & & & & & & & 5 & $100 \%$ & \\
\hline
\end{tabular}

Multiple Linear Regression (MLR). The MLR algorithm establishes the model that describes the relationship between sensor signals and odor scores. All variables used in the models are significant at the 0.01 level. The sample data (105 pork samples from 7 storage time) were separated randomly into two groups: one group for the calibration set was used to develop the calibration models (70 pork samples, 10 samples each day) and the other group containing the remaining samples was used for the prediction set (35 pork samples, 5 samples each day).

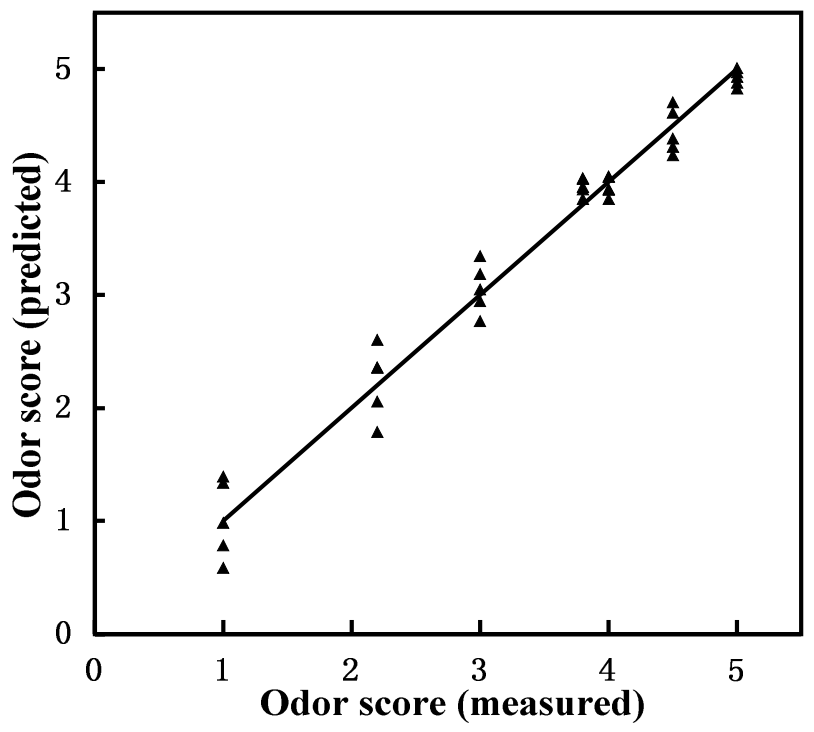

Fig. 5. Prediction of odor scores by MLR model 
The predictive model for the odor score is given below.

$$
\begin{gathered}
S=6.695285+1.314430 X_{1}-0.552935 X_{2}+2.401326 X_{3}-4.553837 X_{4}+ \\
3.023900 X_{5}-2.250302 X_{6}-6.150372 X_{7}-1.827269 X_{8}-1.609080 X_{9}+ \\
8.197955 X_{10},
\end{gathered}
$$

where $\mathrm{S}$ is the odor score, while $X_{1}, X_{2}, X_{3}, X_{4}, X_{5}, X_{6}, X_{7}, X_{8}, X_{9}$ and $X_{10}$ represent the 60th s signal data of the ten sensors MOS1 - MOS10, respectively. The $\mathrm{R}^{2}$ of this model is 0.9848 .

Fig. 5 shows the prediction ability of the e-nose, where each triangle represents the predicted values versus the real value of each measurement. The figures illustrate a linear correlation between the response of sensors and odor scores, indicating that the responses of sensors linearly correlated with the odor scores.

\section{Conclusions}

A PEN2 e-nose detection combined with sensory evaluation were conducted for discrimination and prediction of pork freshness. A pre-experiment was conducted to observe if the sample mass and headspace-generation time would affect the performance of e-nose, and the optimum experimental parameters (10 g sample mass with 5 min headspace-generation time in $500 \mathrm{~mL}$ vial volume) were determined by MANOVA and ANOVA. The later e-nose experiment was taken under such parameters. LDA, BPNN and MLR were employed to observe if the e-nose can classify the pork samples stored during 0-6 d well, as well as to predict the storage time and sensory scores. The results were good: The pork samples could be well discriminated by LDA; $97.14 \% \%$ of the predicting set (with $95.71 \%$ of the training set) was classified correctly using BPNN model; and the MLR model also provided an accurate quality index model between enose signals and sensory scores with high correlation coefficients $\left(R^{2}=0.9848\right)$. These results prove that the e-nose has the potential of being a reliable instrument for the assessment and prediction of pork freshness.

Acknowledgments. The authors acknowledge the financial support of the Chinese National Foundation of Nature and Science through Project 31071548, the Research Fund for the Doctoral Program of Chinese National Higher Education through Project 20100101110133, the Fundamental Research Funds for the Central Universities and the Seed Fund Project on Cross Research For Young Teachers of Zhejiang University.

\section{References}

1. Kong, B.H., Ma, L.Z.: Meat Science and Technology (肉品科学与技术). Chinese Light Industry Press, Beijing (2003)

2. Gram, L., Ravn, L., Rasch, M., Bruhn, J.B., Christensen, A.B., Givskov, M.: Food Spoilage-Interactions Between Food Spoilage Bacteria. Int. J. Food Microbiol. 78(1-2), 79-97 (2002) 
3. Archer, D.L.: The Validation of Rapid Methods In Food Microbiology. Food Control 7, 3-4 (1996)

4. Ellis, D.I., Broadhurst, D., Goodacre, R.: Rapid and Quantitative Detection of The Microbial Spoilage of Beef by Fourier Transform Infrared Spectroscopy and Machine Learning. Anal. Chim. Acta. 514(2), 193-201 (2004)

5. Shirsat, N., Brunton, N.P., Lyng, J.G., McKenna, B., Scannell, A.: Texture, Colour and Sensory Evaluation of A Conventionally and Ohmically Cooked Meat Emulsion Batter. J. Sci. Food Agric. 84(14), 1861-1870 (2004)

6. Malle, P., Vanelle, A.M., Petit, A.: Total Volatile Basic Nitrogen Rates In Salt-Water Fish Muscle. Recueil de Med.Veterinaire 165(4), 395-402 (1989)

7. FDA-US Food \& Drugs Administration: Bacteriological Analytical Manual, 8th edn. AOAC International, Gaithersburg (1998)

8. Limbo, S., Sinelli, N., Torri, L., Riva, M.: Freshness Decay and Shelf Life Predictive Modelling of European Sea Bass (Dicentrarchus Labrax) Applying Chemical Methods and Electronic Nose. LWT Food Sci. Technol. 42(5), 977-984 (2009)

9. Gardner, J.W., Bartlett, P.N.: A Brief History of Electronic Nose. Sens. Actuators, B 18(19), 211-220 (1994)

10. Yu, Y., Wang, J., Zhou, M.: Research Developments of Electronic Nose and Its Application in Processing of Agriculture Products. Journal of Zhejiang University: Agriculture \& Life Science 29(5), 579-584 (2003) (in Chinese)

11. García, M., Aleixandre, M., Gutiérrez, J., Horrillo, M.C.: Electronic Nose For Wine Discrimination. Sens. Actuators, B 113(2), 911-916 (2006)

12. Torri, L., Sinelli, N., Limbo, S.: Shelf Life Evaluation of Fresh-Cut Pineapple by Using An Electronic Nose. Postharvest Biol. Technol. 56(3), 239-245 (2010)

13. Method for analysis of hygienic standard of meat and meat products. National Standard of the People's Republic of China: GB/T 5009.44-2003 (2003)

14. Olsson, J., Börjesson, T., Lundstedt, T., Schnürer, J.: Detection and Quantification of Ochratoxin A and Deoxynivalenol In Barley Grains by GC-MS and Electronic Nose. Int. J. Food Microbiol. 72, 203-214 (2002)

15. Buratti, S., Benedetti, S., Scampicchio, M., Pangerod, E.C.: Characterization and Classification of Italian Barbera Wines by Using An Electronic Nose and An Amperometric Electronic Tongue. Anal Chim Acta, 133-139 (2004)

16. Neely, K., Taylor, C., Prosser, O., Hamlyn, P.F.: Assessment of Cooked Alpaca and Llama Meats From the Statistical Analysis of Data Collected Using An Electronic Nose. Meat Sci. 58(1), 53-58 (2001)

17. Magana, N., Evans, P.: Volatiles As An Indicator of Fungal Activity and Differentiation Between Species, and The Potential Use of Electronic Nose Technology For Early Detection of Grain Spoilage. J. Stored Prod. Res. 36(4), 319-340 (2000)

18. Zhang, Q.Y., Zhang, S.P., Xie, C.S., Zeng, D.W., Fan, C.Q., Li, D.F., et al.: Characterization of Chinese Vinegars by Electronic Nose. Sens. Actuators, B 119(2), 538-546 (2006)

19. Pang, L.J., Wang, J., Lu, X., Yu, H.: Discrimination of Storage Age for Wheat by E-nose. Transactions of the ASABE 51(5), 1707-1712 (2008)

20. Muthukrishnan, N., Paulo Davim, J.: Optimization of Machining Parameters of Al/SiCMMC with ANOVA and ANN Analysis. J. Mater. Process Tech. 209(1), 225-232 (2009)

21. Wikipedia, http://en.wikipedia.org/wiki/MANOVA\#cite_note-0

22. Backhoff, E., Larrazolo, N., Rosas, M.: The Level of Difficulty and Discrimination Power of The Basic Knowledge and Skills Examination (EXHCOBA). Revista Electrónica de Investigación Educativa 2(1),

http://redie.uabc.mx/vol2nol/contents-backhoff.html 